\title{
Machine Learning Classification Techniques for Detecting the Impact of Human Resources Outcomes on Commercial Banks Performance
}

\author{
Sulaiman O. Atiku $\mathbb{D}^{1}$ and Ibidun C. Obagbuwa $\mathbb{D}^{2}$ \\ ${ }^{1}$ Harold Pupkewitz Graduate School of Business, Namibia University of Science and Technology, Windhoek, Namibia \\ ${ }^{2}$ Department of Computer Science and Information Technology, Sol Plaatje University, Kimberley, South Africa \\ Correspondence should be addressed to Ibidun C. Obagbuwa; ibidun.obagbuwa@spu.ac.za
}

Received 7 May 2021; Accepted 30 July 2021; Published 21 September 2021

Academic Editor: Ridha Ejbali

Copyright (c) 2021 Sulaiman O. Atiku and Ibidun C. Obagbuwa. This is an open access article distributed under the Creative Commons Attribution License, which permits unrestricted use, distribution, and reproduction in any medium, provided the original work is properly cited.

\begin{abstract}
The banking industry is a market with great competition and dynamism where organizational performance becomes paramount. Different indicators can be used to measure organizational performance and sustain competitive advantage in a global marketplace. The execution of the performance indicators is usually achieved through human resources, which stand as the core element in sustaining the organization in the highly competitive marketplace. It becomes essential to effectively manage human resources strategically and align its strategies with organizational strategies. We adopted a survey research design using a quantitative approach, distributing a structured questionnaire to 305 respondents utilizing efficient sampling techniques. The prediction of bank performance is very crucial since bad performance can result in serious problems for the bank and society, such as bankruptcy and negative influence on the country's economy. Most researchers in the past adopted traditional statistics to build prediction models; however, due to the efficiency of machine learning algorithms, a lot of researchers now apply various machine learning algorithms to various fields, including performance prediction systems. In this study, eight different machine learning algorithms were employed to build performance models to predict the prospective performance of commercial banks in Nigeria based on human resources outcomes (employee skills, attitude, and behavior) through the Python software tool with machine learning libraries and packages. The results of the analysis clearly show that human resources outcomes are crucial in achieving organizational performance, and the models built from the eight machine learning classifier algorithms in this study predict the bank performance as superior with the accuracies of $74-81 \%$. The feature importance was computed with the package in Scikitlearn to show comparative importance or contribution of each feature in the prediction, and employee attitude is rated far more than other features. Nigeria's bank industry should focus more on employee attitude so that the performance can be improved to outstanding class from the current superior class.
\end{abstract}

\section{Introduction}

Today's business environment is highly competitive and changing rapidly in terms of globalization and technology innovations, and it becomes imperative to develop the internal potential by paying adequate attention to people management and the workforce that enables the systems to operate. Hence, human resources management has been considered vital in obtaining a sustainable competitive advantage in the face of globalization and advances in technology [1-6]. There are drastic changes in the banking sector due to the application of electronic banking technologies, where financial institutions are now making use of the World Wide Web and other appropriate technologies and software to process applications for various products at a minimum time and cost $[7,8]$. This has greatly improved bank operations and services across the globe.

The traditional data analysis techniques are unable to cope with large volumes of data, and there is an increase in the huge volume of data in day-to-day operations from 
internal and external sources. The traditional data analysis does not effectively harness the increased processing power. Predictable variables independence limits their application in the real world, and these methods may be theoretically invalid for the finite sample which can pose a problem in applying it to the performance prediction because the multivariate-normality assumption for the independent variable is frequently violated in financial datasets [9-11].

In a financial institution, data are the assets. Therefore, the value of the data can be evaluated when the organization can extract the valuable knowledge hidden in the raw data. Data mining involves the extraction of interesting patterns from raw data using statistical and machine learning techniques [7]. Data mining techniques can be used to build an excellent predictive model and visualize its report into meaningful information [7]. The data mining techniques and tools can predict the future trend and behavior of a system and discover the previous unknown patterns [7-9].

Previous works on the impact of human resources on organizational performance were analyzed using descriptive and inferential statistics. Antwi et al. adopted descriptive statistics and multivariate regression for analyzing data [3]. Delery and Gupta adopted descriptive statistics and hierarchical regression analysis to determine the influence of human resources on organizational performance [4]. Atiku et al. used both descriptive and inferential statistics to determine the influence of human resource outcomes on bank performance [6]. Descriptive statistics was extensively used to analyze data [1, 12-14]. In this study, we adopted data mining technology in detecting and predicting the influence of human resources outcomes-employee skill, attitude, and behavior on bank performance. By adopting data mining technology, an organization can access the right information timely from the huge volume of raw data. Data mining techniques can discover hidden patterns that may not be discovered by traditional data analysis.

To the best of our knowledge, one of the recent studies carried out a survey on the relationship between organizational capabilities using big data predictive analytics while achieving superior organizational performance. The result of their findings shows that financial institutions need to adopt green and flexible technologies to achieve higher operational performance which enhances overall profit. Moreover, few studies have been conducted on the application of data mining to the banking sector in the areas such as customer retention, automatic credit approval, fraud detection, marketing, and risk management $[7-10,15,16]$, but none on the prediction of bank performance concerning human resources outcomes using the data mining approach.

The remaining part of this study is organized as follows: Section 2 shows the methodology used in this study. Section 3 presents the experiments conducted and the results of the experiments. In Section 4, conclusions are drawn with the recommendation for future research.

\section{Materials and Methods}

The CRISP-DM model was implemented in this work. The model has been widely used by many researchers in the last decade [16-19]. CRISP-DM is a nonpropriety, freely available, and cross-industry standard for data mining projects. It is a cyclic method comprising of six phases: business understanding, data understanding, data preparation, modeling, evaluation, and deployment as depicted in Figure 1 [20]. Python was used as a software tool for implementing this work. It is open-source software that can offer a wide range of data mining classification techniques.

At the business understanding phase, a critical and extensive review of the literature was carried out to study the existing problems in the bank that have been handled by the data mining approach. The study employed a survey approach to investigate the impact of human resource outcomes on organizational performance. Multiple methods were adopted for gathering the data via the use of a structured questionnaire, personal interviews, observations, and other documented evidence (annual reports/statements of accounts for three consecutive years) [21]. The business problem was first identified which is the growing need of the commercial banks in Nigeria to know that human resource outcomes (employee skills, attitude, and behavior) have a great impact on the performance of the bank using a data mining approach to extract all hidden facts and make a prediction. The performance of the bank is predicted to be able to approach the competitive market campaigns exactly those human resource outcomes that indicate good performance of the organization. The identified problem was transformed into a data mining task by classifying organization performance into five categories which are outstanding, superior, good, average, and poor (Table 1), and by analyzing the available employee data with the data mining techniques that are selected for the classification. This is considered a supervised learning task since the classification models are built from the data that have a known target variable.

At the data understanding phase, the employee data were studied to understand the types of data collected from the bank employees that are stored in an electronic database. The rules and procedures for the collection and storage of the bank employee data were also reviewed. The details are provided in the study by Atiku [21].

At the data preprocessing phase, employee data from the employee database were extracted and organized. This contains data for 305 employees, described by 19 parameters such as sex, marital status, department, educational qualification, work experience, organizational culture, organizational learning culture, organizational performance, employee attitudes, and so on [21]. The available data were transformed, and some of the parameters were removed. Only the parameter (organization performance (Table 2) and human resources outcomes (Table 3)) that are important for the research were processed. The challenge in this data mining project is essential to predict bank performance based on human resource outcomes. Organization performance is the selected target variable to be learned by the data mining algorithm. A categorical target variable is designed using five values (categories) in Table 1. The bank performance can be outstanding, superior, good, average, and poor. For each of the six performances given 


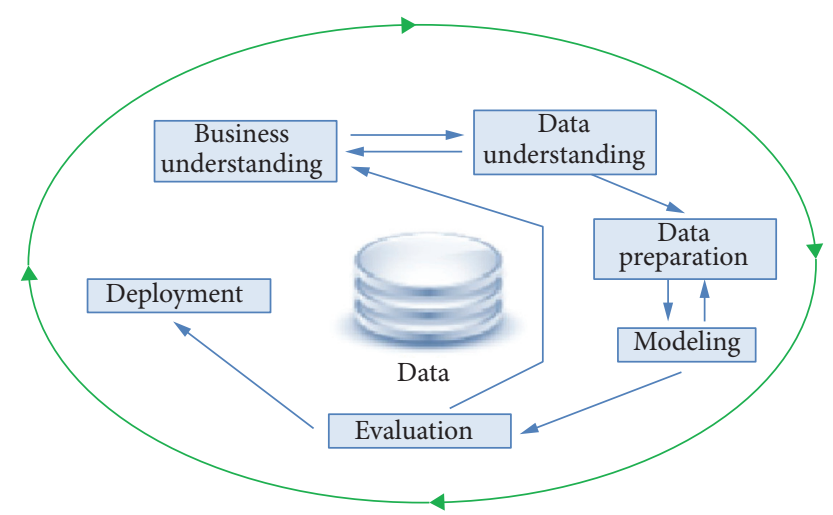

FIgURE 1: The cross-industry standard process for data mining (CRISP-DM) [20].

TABLE 1: Bank performance grading scale.

\begin{tabular}{lccc}
\hline S/N & Rating & Range (\%) & Description \\
\hline 1 & Rating 1 & $91-100$ & Outstanding \\
2 & Rating 2 & $71-90$ & Superior \\
3 & Rating 3 & $51-70$ & Good \\
4 & Rating 4 & $31-50$ & Average \\
5 & Rating 5 & $<30$ & Poor \\
\hline
\end{tabular}

TABLE 2: Organization performance framework.

\begin{tabular}{lr}
\hline $\mathrm{S} / \mathrm{N}$ & Items \\
\hline 1 & In recent years, the change in competitive advantage relative to the largest competitor has markedly improved \\
2 & In recent years, the change in market share relative to the largest competitor has markedly improved \\
3 & In recent years, the change in profit relative to the largest competitor has markedly improved \\
4 & In recent years, change in cost (product or services) relative to the largest competitor has reduced \\
5 & In recent years, the change in sales revenue relative to the largest competitor has greatly increased \\
6 & In recent years, the change in customer satisfaction relative to the largest competitor has greatly increased \\
\hline
\end{tabular}

TABLE 3: Human resources outcomes framework dimension code and items.

\begin{tabular}{|c|c|c|c|}
\hline $\begin{array}{l}\mathrm{S} / \\
\mathrm{N}\end{array}$ & Items & Dimension & $\begin{array}{l}\text { Dimension } \\
\text { code }\end{array}$ \\
\hline 1 & Sufficient effort is made to get the opinions of people who work here & \multirow{12}{*}{$\begin{array}{l}\text { Employee } \\
\text { attitude }\end{array}$} & \multirow{12}{*}{ EmpAtt } \\
\hline 2 & I am given a real opportunity to improve my skills in this company & & \\
\hline 3 & I feel encouraged to come up with new and better ways of doing things & & \\
\hline 4 & I like the kind of job I do & & \\
\hline 5 & My work gives me a feeling of personal accomplishment & & \\
\hline 6 & My job makes good use of my skills and abilities & & \\
\hline 7 & How satisfied are you with your involvement in the decisions that affect your work? & & \\
\hline 8 & How satisfied are you with the opportunity to get a better job at this company? & & \\
\hline 9 & $\begin{array}{c}\text { How satisfied are you with the information you receive from management regarding what is going } \\
\text { on in this company? }\end{array}$ & & \\
\hline 10 & How satisfied are you with the training you received for your present job? & & \\
\hline 11 & How satisfied are you with your physical working conditions? & & \\
\hline 12 & How satisfied are you with your involvement in the decisions that affect your work? & & \\
\hline
\end{tabular}


TABLE 3: Continued.

\begin{tabular}{|c|c|c|c|}
\hline $\begin{array}{l}\mathrm{S} / \\
\mathrm{N}\end{array}$ & Items & Dimension & $\begin{array}{l}\text { Dimension } \\
\text { code }\end{array}$ \\
\hline 13 & I participate in solving problems in the organization & \multirow{18}{*}{$\begin{array}{l}\text { Employee } \\
\text { behavior }\end{array}$} & \multirow{18}{*}{ EmpBeh } \\
\hline 14 & I become involved in work committees in the organization & & \\
\hline 15 & The manager proposes changes that will have an impact outside his/her workgroup & & \\
\hline 16 & The manager expresses his/her ideas in discussion groups in the organization & & \\
\hline 17 & The manager makes suggestions to improve the organization’s functioning & & \\
\hline 18 & I help coworkers do their work & & \\
\hline 19 & I provide constructive feedback that helps a coworker & & \\
\hline 20 & I keep coworkers informed of the progress of his/her work in group projects & & \\
\hline 21 & Questions inefficient ways of doing things in his/her workgroup & & \\
\hline 22 & Introduces new ways of doing things in his/her workgroup & & \\
\hline 23 & Suggests improvements to increase his/her work group's efficiency & & \\
\hline 24 & $\begin{array}{c}\text { An employee does everything in his/her power to satisfy the customer, even when there are } \\
\text { problems }\end{array}$ & & \\
\hline 25 & Makes suggestions to improve the products and/or services offered to customers & & \\
\hline 26 & Projects a positive image of the organization to customers & & \\
\hline 27 & The supervisor helps you by doing things that are not part of his/her regular duties & & \\
\hline 28 & The supervisor keeps you informed of important events which concern you & & \\
\hline 29 & The supervisor suggests ways towards improving the work group's performance & & \\
\hline 30 & The supervisor advises you on ways to improve your management practices & & \\
\hline 31 & Ability to attract the best employees & \multirow{12}{*}{ Employee skills } & \multirow{12}{*}{ EmpSkills } \\
\hline 32 & Ability to retain essential employees & & \\
\hline 33 & Cooperation between management and other employees & & \\
\hline 34 & Cooperation among employees in general & & \\
\hline 35 & Motivation among employees in general & & \\
\hline 36 & Quality consciousness among employees in general & & \\
\hline 37 & Spending per employee & & \\
\hline 38 & Absence rate & & \\
\hline 39 & Turnover rate & & \\
\hline 40 & Job satisfaction & & \\
\hline 41 & Organizational commitment & & \\
\hline 42 & Customer complaints & & \\
\hline
\end{tabular}

in Table 2, the respondent scores (1-4) were used [21]. Table 1 depicts the scaled performance rating which was performed and adjusted to suit banking performance based on the Fitch recovery rating [22]. One of the three nationally acceptable ratings by the US security and exchange commission in 1975 is the Fitch Rating Inc. Since the classification technique label must be categorical, scaling is important.

At the modeling phase, the data mining model is built by classifying bank performance into five categories as given in Table 1. In this work, we employed several classification algorithms that have the potential to yield good results including decision tree, logistic regression, nearest neighbor algorithm, random forest, gradient boosting, support vector machine, ensemble [23-25], and deep learning. Scikit-learn-Python package for machine learning was used to design the experiments of the proposed models. The selected classification algorithms used for this data mining project were applied to the final dataset consisting of 305 bank employee records with 4 attributes. For implementation, the 4 attributes and descriptions are depicted in Table 4 . The results of the work are given in Section 4.
TABLE 4: Description of features.

\begin{tabular}{lccc}
\hline Feature number & Feature code & Data type & Description \\
\hline 1 & EmpAtt & Numeric & Predictive \\
2 & EmpBeh & Numeric & Predictive \\
3 & EmpSkills & Numeric & Predictive \\
4 & Performance & Categorical & Class \\
\hline
\end{tabular}

\section{Experiments, Results, and Discussion}

The main objective of this study is to detect the possibility of predicting the class (output) variable with the input variables that are retained in the bank performance model. In our experiments, we used the most common classification techniques from Scikit-learn. These classification techniques are described in Section 3.1 of this study. The percentage split approach was used for the dataset to divide it into training and test sets $(70 \%$ for the training dataset and $30 \%$ for the test dataset). The performance of the classification models was measured in our experiments on the input features using a confusion matrix from Scikit-learn to evaluate records that are correctly or incorrectly predicted by the classifiers. A confusion matrix is an $\mathrm{N} \times \mathrm{N}$ table that summarizes how 
successful a classification model's predictions were, that is, the correlation between the label and the model's classification. One axis of a confusion matrix is the label that the model predicted and the other axis is the actual label. $N$ represents the number of classes. In a binary classification problem, $N=2$. For instance, Figure 2 is a sample confusion matrix for a binary classification problem. In this work, as depicted in Table 1, there are 5 output classes for bank performance (outstanding, superior, good, average, and poor). A confusion matrix is a table with 4 different combinations of predicted and actual values as illustrated in Figure 2. The quantities, true positive (TP), false positive (FP), true negative (TN), and false negative (FN) are associated with the confusion matrix. The confusion matrix is exceedingly useful for measuring recall, precision, specificity, and accuracy as expressed in equations (1)-(6). In this study, the following metrics (equations (1)-(6)) were used to measure the performance of the classifiers.

(i) Sensitivity/true positive rate/recall is given as

$$
\text { Sensitivity }=\frac{\mathrm{TP}}{\mathrm{TP}+\mathrm{FN}} \text {. }
$$

Sensitivity represents the percentage of the positive class that is correctly classified.

(ii) False negative rate is given as

$$
\mathrm{FNR}=\frac{\mathrm{FN}}{\mathrm{TP}+\mathrm{FN}} \text {. }
$$

False negative rate (FNR) represents the percentage of the positive class that is incorrectly classified by the classifier.

(iii) Specificity/true negative rate is given as

$$
\text { Sensitivity }=\frac{\mathrm{TN}}{\mathrm{TN}+\mathrm{FP}} \text {. }
$$

Specificity represents the percentage of the negative class that is correctly classified.

(iv) False positive rate is given as

$$
\mathrm{FPR}=\frac{\mathrm{FP}}{\mathrm{TN}+\mathrm{FP}}=1-\text { specificity. }
$$

FPR represents the percentage of the negative class that is incorrectly classified by the classifier.

(v) Accuracy (ACC) is given as

$$
\mathrm{ACC}=\frac{(\mathrm{TP}+\mathrm{TN})}{(\mathrm{TP}+\mathrm{TN}+\mathrm{FP}+\mathrm{FN})} .
$$

ACC is the percentage of the total features that are correct.

(vi) $F$-score (F1) or $F$-measure is given as

$$
F 1=\frac{2 * \text { precision } * \text { recall }}{(\text { precision }+ \text { recall })} \text {. }
$$

$F 1$ is a weighted harmonic average of precision and recall.
Sensitivity and specificity are the most important out of the metrics shown above; they show the proportions that are correctly classified either as positive or negative. Sensitivity, specificity, accuracy, and F-score (F1) among other metrics were used for assessing the classifiers considered in this work. Two types of errors, namely, type 1 and type 2 do occur while evaluating the performance of machine learning classifiers:

Type 1 error: false positive (FP). This type of error occurs because the predicted value was falsely predicted. The actual value was negative, but the model predicted a positive value (equation (4)).

Type 2 error: false negative (FN). The predicted value was falsely predicted. The actual value was positive, but the model predicted a negative value (equation (2)).

The visualization of the impact of the three features of human resources outcome (employee attitudes, behavior, and skills) is shown in Figures 3(a)-3(c), respectively. The three selected features show a greater percentage for superior performance. The bank performance is generally superior on the input features.

3.1. Machine Learning Algorithms. Machine learning (ML) is a branch of artificial intelligence for building models/systems that can learn from data. ML is the process of teaching a computer system how to make accurate predictions when fed with data. ML algorithms are the engines of machine learning (i.e., it is the algorithm that turn datasets into models). These algorithms consist of a target/outcome variable (or dependent variable) which is to be predicted from a given set of predictors (independent variables). A function that maps inputs to desired outputs is created by using the set of variables. The procedure as shown in Figure 4 continues until the model accomplishes an anticipated level of accuracy on the training dataset. The ML algorithms described in the subsections 3.1.1-3.1.8 were used for classification in this study.

3.1.1. K-Nearest Neighbors Classifier. The K-nearest neighbors classifier (K-NN) algorithm is arguably the simplest machine learning algorithm. Building the model consists only of storing the training dataset. To predict a new data point, the algorithm finds the closest data points in the training dataset - its "nearest neighbors." The K-NN algorithm is for both classification and regression but is largely used for classification. It is a supervised learning algorithm that deems various centroids and utilizes the Euclidean function for distance comparison. It analyses results and classifies each point to the group with all closest points. New instances are classified using a majority vote of $k$ of its neighbors, and the instance is allocated to a class that is most common among its $K$-nearest neighbors [19]. The basic steps for K-NN are listed as follows and depicted in Figure 5. Basic steps for K-NN are as follows.

(i) Calculate distance

(ii) Find closest neighbors

(iii) Vote for labels 


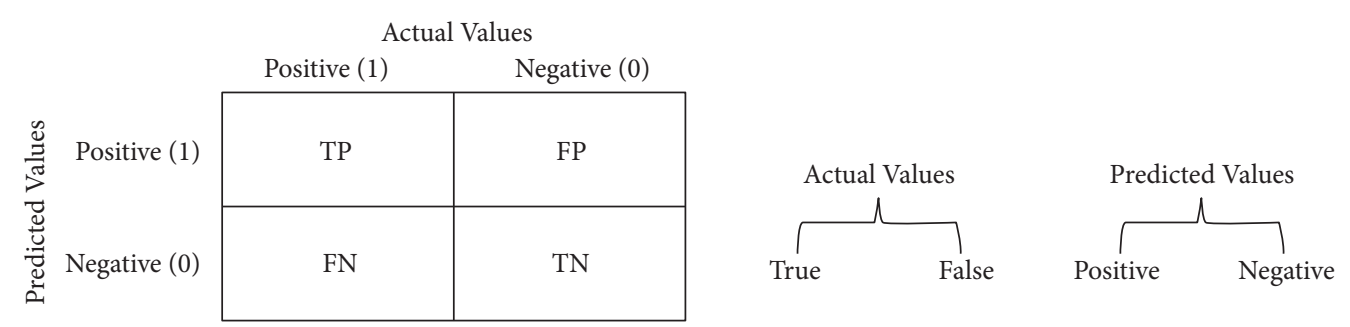

Figure 2: Confusion matrix.

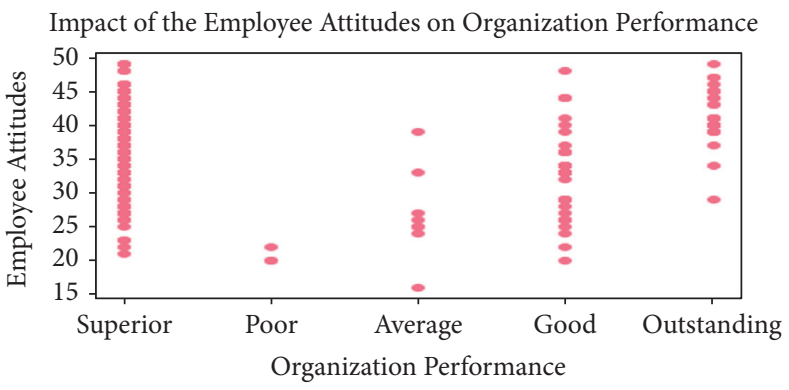

(a)

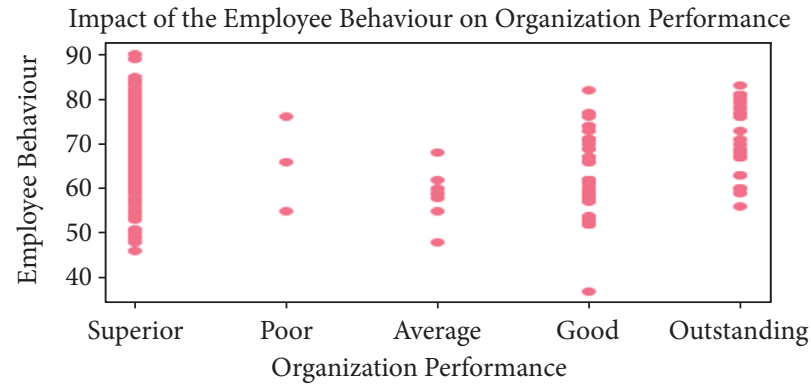

(b)

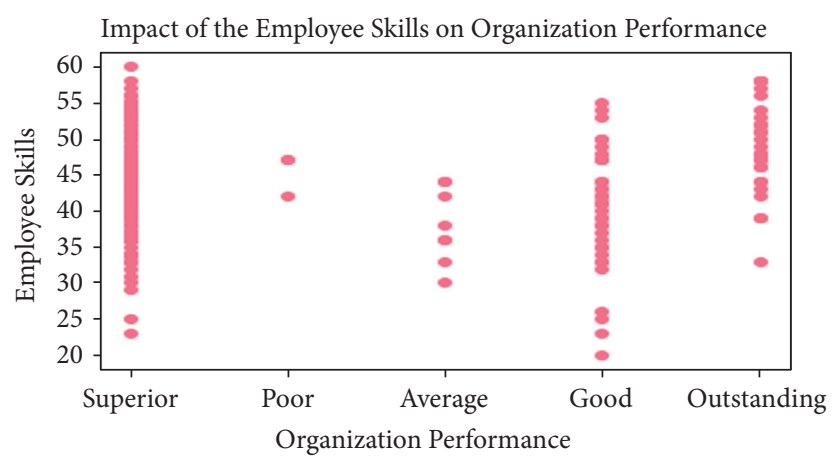

(c)

Figure 3: Impact of human resource outcomes (employee attitude, behavior, and skills) on bank industry performance. (a) Employees attitude. (b) Employees behavior. (c) Employees skills.

In this study, we build a classification model using the K-NN algorithm. Figure 6 depicts the result of the experiments on training and test datasets with the accuracies of $74 \%$ and $75 \%$, respectively.

Accuracy of the K-NN classifier on the training set is 0.74 . The accuracy of the K-NN classifier on the test set is 0.75 .

Predict by choosing K-NN to model with $k=100$, after seeing that a value of $k=100$ is a pretty good number of neighbors for this model. This was used to fit the model for the entire dataset instead of just the training set. An example of an out-of-sample observation of prediction in this work is given as follows: knn.predict ([[88, 44, 50]]), where $88,44,50$ is the sample employee' attitude, behavior, and skills respectively; and the output is array(("Superior"), dty$\mathrm{pe}=\mathrm{object})$. The bank performance is predicted to be "Superior," i.e., it shows a superior performance class for the three input variables. We evaluate the performance of the K-NN classifier by computing the confusion matrix shown in Figure 7. The K-NN algorithm classified the bank performance as "Superior class." The superior class has the greatest proportion of precision, recall, $F 1$-score, and support (Figure 7). The "support" in Figure 7 is the number of true response samples in a class.

3.1.2. Logistic Regression. Logistic regression is appropriate for binary classification, although called regression. It is essentially a classification algorithm that fits data into a logistic function. It is an exceptional machine learning algorithm that estimates discrete values such as $0 / 1$, yes/no, and true/false based on a given set of independent variables and uses a logistic function to predict the likelihood of an event, of which its output is between 0 and 1 as shown in Figure 8.

In our experiment with training and test datasets, we obtained accuracies of $74.6 \%$ and $76.1 \%$, respectively. Training set' accuracy is 0.746 . The test set' accuracy is 0.761 . 


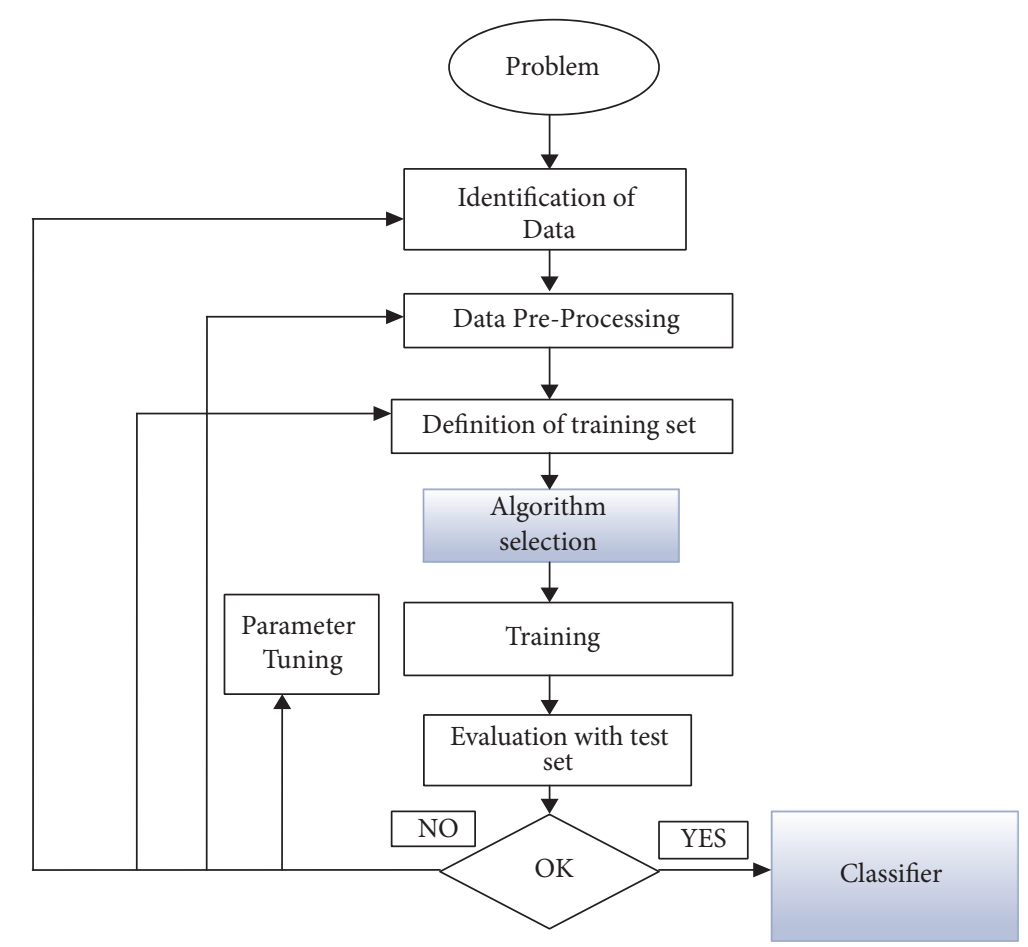

Figure 4: Machine learning classification process [26].

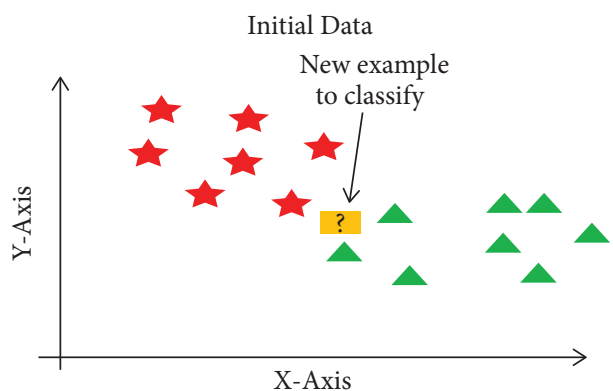

Class A Class B

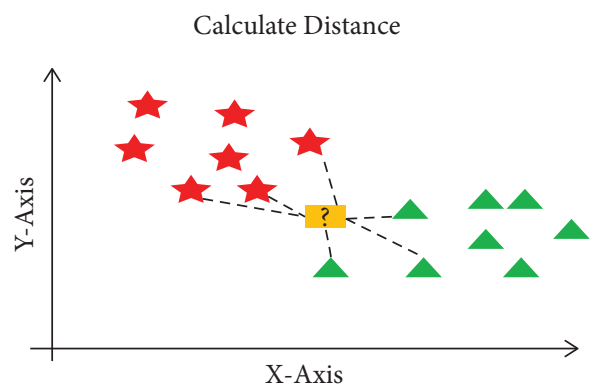

Class A

Class B

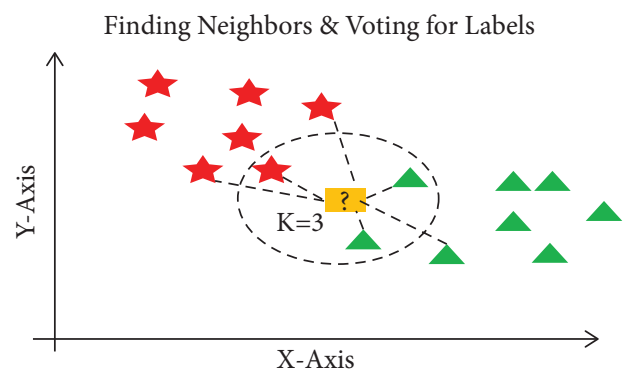

Class A

Class B

FIGURE 5: K-NN: https://www.datacamp.com/community/tutorials/k-nearest-neighbor-classification-scikit-learn.

Experimenting with the entire dataset, we obtain an accuracy of $75 \%$ as shown in Figure 9, confusion matrix, and precision computation. The result also confirms that the bank performance is classified as "superior" with an accuracy of $75 \%$.
3.1.3. Decision Tree Classifier. The decision tree algorithm works on both categorical and continuous dependent variables, even though usually used for classification. Models built with a decision tree algorithm take an instance and 


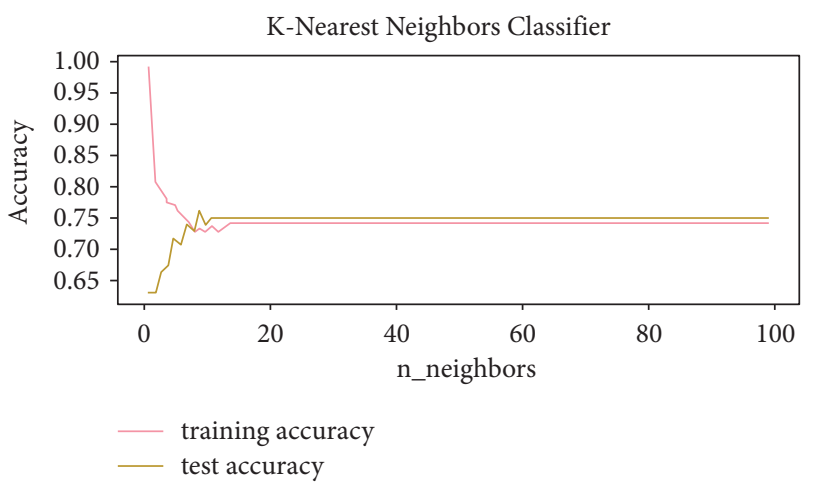

FIGURE 6: K-NN computation for training and test datasets.

\begin{tabular}{|c|c|c|c|c|c|c|}
\hline$\left[\begin{array}{ll}{[} & 0\end{array}\right.$ & 0 & 3 & $0]$ & & & \\
\hline 0 & 0 & 8 & $0]$ & & & \\
\hline 0 & 0 & 29 & $0]$ & & & \\
\hline 0 & 0 & $0 \quad 227$ & $0]$ & & & \\
\hline 0 & 0 & 38 & 0] ] & & & \\
\hline & & & ecision & recall & fl-score & support \\
\hline & Poor & & 0.00 & 0.00 & 0.00 & 3 \\
\hline Ave & erage & & 0.00 & 0.00 & 0.00 & 8 \\
\hline Outstan & hding & & 0.00 & 0.00 & 0.00 & 29 \\
\hline Supe & erior & & 0.74 & 1.00 & 0.85 & 227 \\
\hline & good & & 0.00 & 0.00 & 0.00 & 38 \\
\hline $\mathrm{accu}$ & גracy & & & & 0.74 & 305 \\
\hline macro & avg & & 0.15 & 0.20 & 0.17 & 305 \\
\hline weighteo & d avg & & 0.55 & 0.74 & 0.64 & 305 \\
\hline
\end{tabular}

Figure 7: K-NN confusion matrix and accuracy results.

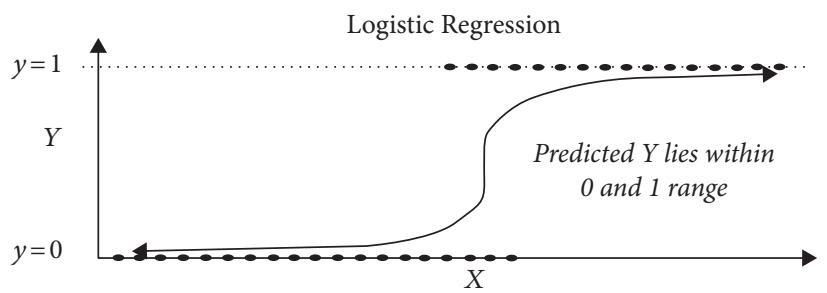

FIGURE 8: Logistic regression: https://www.datacamp.com/community/tutorials/logistic-regression-R.

traverse the tree; based on the conditional statement, it compares the essential features and determines if it descends the left $\backslash$ right branch based on the result. Normally, critical features are nearer to the root (Figure 10(a)).

Basis steps in decision tree are as follows:

(i) Select the best attribute using attribute selection measures (ASM) to split the records

(ii) Make that attribute a decision node and breaks the dataset into smaller subsets

(iii) Starts tree building by repeating this process recursively for each child until one of the conditions will match:

(i) All the tuples belong to the same attribute value (ii) There are no more remaining attributes

(iii) There are no more instances

The decision tree generation (Figure 10(b)) illustrates how decision tree models are built and tested from a dataset that is being split into training and test data. The training data is used for building models while the test data is for evaluating the model. The performance evaluation of the built model is performed by using some metrics (Equations (1)-(6)). Figure 11 depicts the performance evaluation results of the decision tree classifier of this work. The bank's performance was classified as superior with a precision of $77 \%$.

Our experiment with the decision tree algorithm depicts "superior" bank performance with the accuracy of $77.9 \%$ and $71.7 \%$ for the training and test data, respectively. The 


\begin{tabular}{|c|c|c|c|c|c|}
\hline & & & & & \\
\hline L L & 1 & 0 & 0 & 2 & 0] \\
\hline[ & 0 & 0 & 0 & 8 & $0]$ \\
\hline[ & 0 & 0 & 0 & 29 & $0]$ \\
\hline[ & 0 & 0 & 0 & 227 & $0]$ \\
\hline[ & 0 & 0 & 0 & 38 & $0]$ ] \\
\hline
\end{tabular}

$\begin{array}{rrrrr} & \text { precision } & \text { recall } & \text { fl-score } & \text { support } \\ \text { Poor } & 1.00 & 0.33 & 0.50 & 3 \\ \text { Average } & 0.00 & 0.00 & 0.00 & 8 \\ \text { Outstanding } & 0.00 & 0.00 & 0.00 & 29 \\ \text { Superior } & 0.75 & 1.00 & 0.85 & \mathbf{2 2 7} \\ \text { good } & 0.00 & 0.00 & 0.00 & 38 \\ \text { accuracy } & & & & 305 \\ \text { macro avg } & 0.35 & 0.27 & 0.27 & 305 \\ \text { weighted avg } & 0.57 & 0.75 & 0.64 & 305\end{array}$

FIGURE 9: Logistic regression confusion matrix and accuracy results.

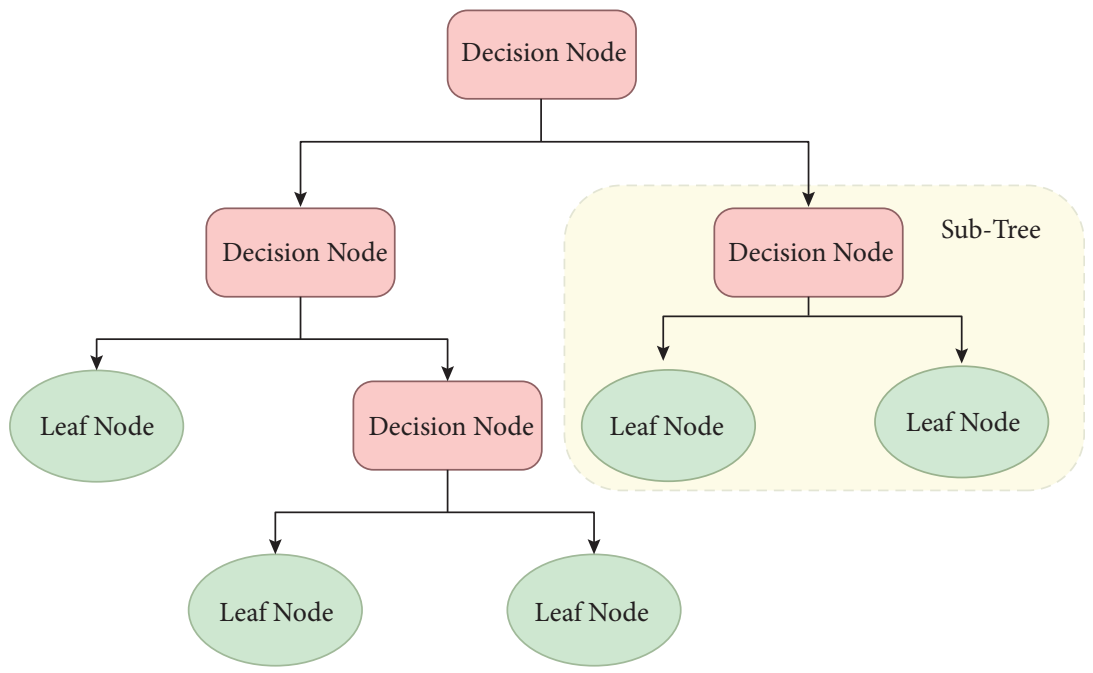

(a)

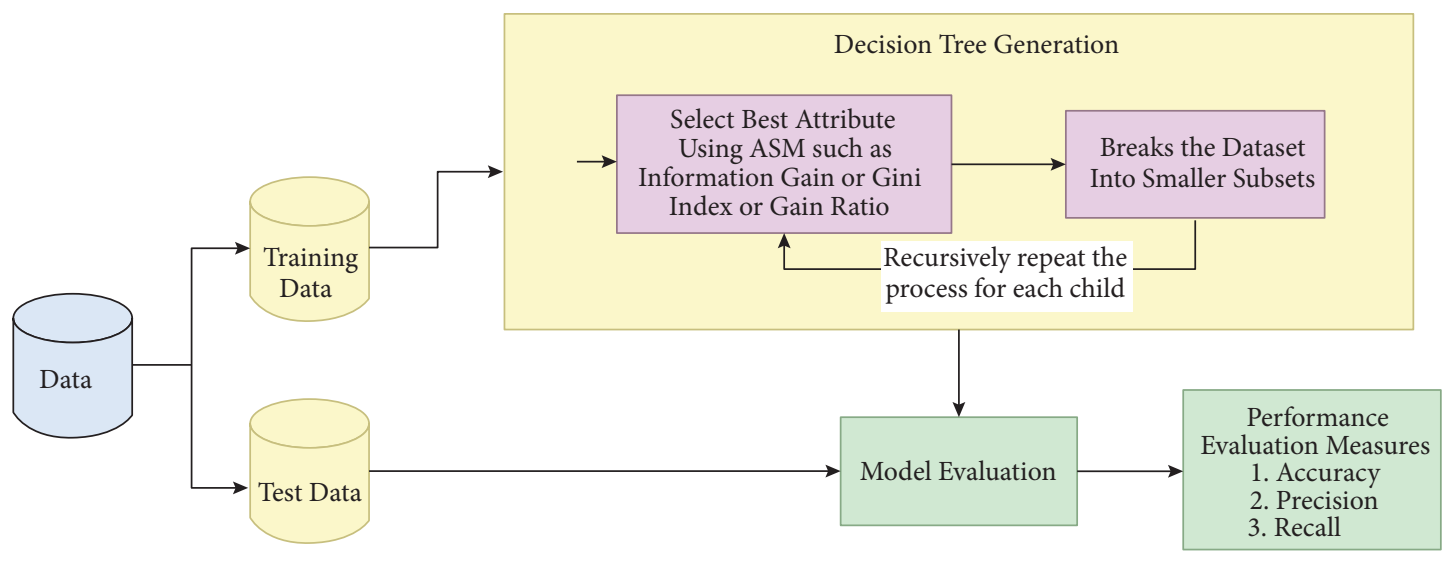

(b)

FIGURE 10: Decision tree and decision tree generation: https:/www.datacamp.com/community/tutorials/decision-tree-classificationpython. (a) Decision tree. (b) Decision tree generation. 


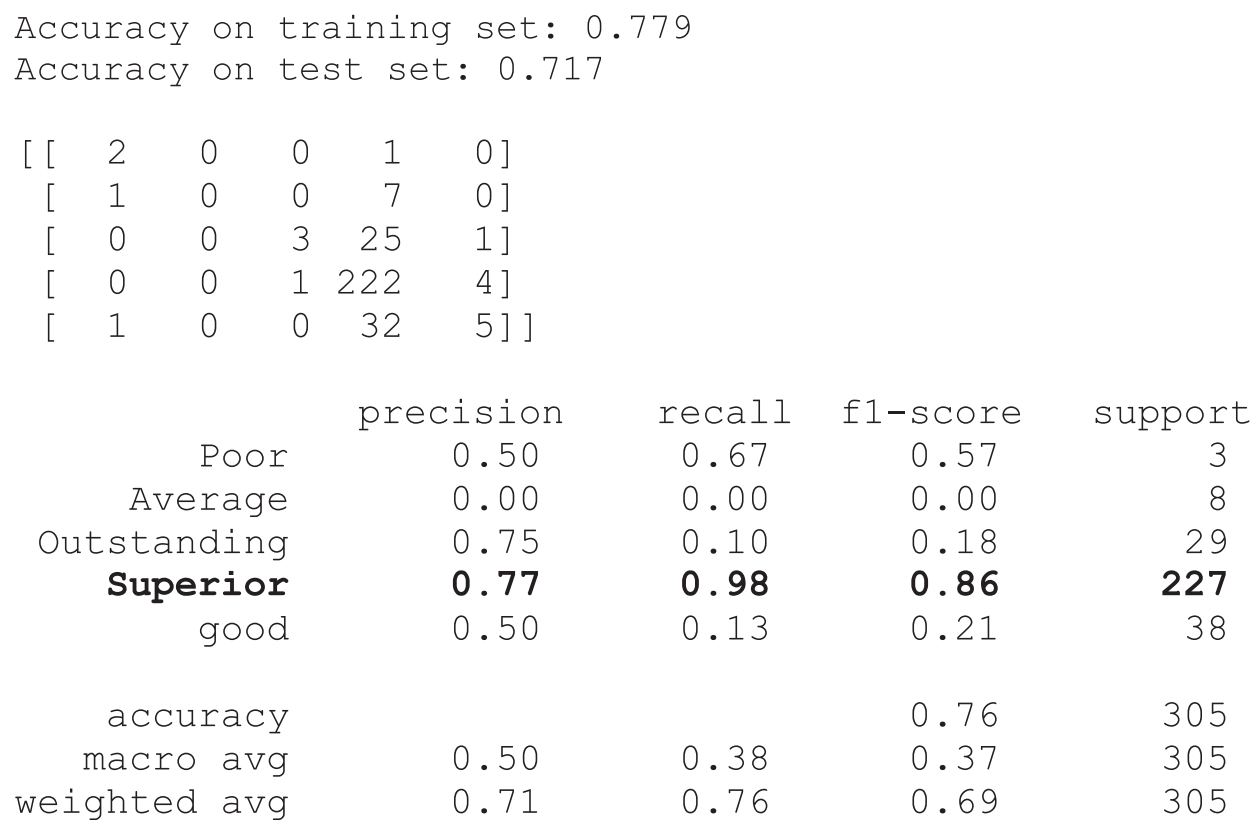

FIGURE 11: Decision tree confusion matrix and accuracy results.

accuracy on the training set is 0.779 . The accuracy on the test set is 0.717 .

3.1.4. Random Forest. A random forest is an ensemble of decision trees that function by building a multitude of decision trees during training time and present the class with the mode classification of the individual trees. The four steps to the random forest are as follow and are also depicted in Figure 12.

Steps are as follows:

(i) Select random samples from a given dataset

(ii) Construct a decision tree for each sample and get a prediction result from each decision tree

(iii) Perform a vote for each predicted result

(iv) Select the prediction result with the most votes as the final prediction

In our experiments, a random forest consisting of 100 trees was applied to the bank dataset, and we obtained $75.1 \%$ and $75.0 \%$ accuracies, respectively, on the training and test datasets. We compute the performance evaluation of the random forest classifier using the confusion matrix and obtained the results as shown in Figure 13. The classifier predicted the bank performance as superior with a precision of $75 \%$.

3.1.5. Gradient Boosting Classifier. Gradient boosting is a machine learning boosting that minimizes the overall prediction errors by combining the previous models to emerge the next possible best model. The target outcome depends on how much changing that prediction influences the overall prediction error. The adaptive boosting method combined with weighted minimization, after which the classifiers and weighted inputs are recalculated is being referred to as gradient boosting classifiers (Figure 14). Gradient boosting classifiers aim to minimize the loss or the difference between the actual class value of the training example and the predicted class value. It operates similar to gradient descent in a neural network.

In our experiment on gradient boosting classifier on the bank dataset, we obtained the accuracy on the training set as $0.817(81.7 \%)$ and on the test set as $0.750(75.0 \%)$.

For the performance evaluation of the classifier by computing the confusion matrix, we arrived at the results shown in Figure 15. The classifier predicts the superior class of bank performance with the $81 \%$ precision.

3.1.6. Support Vector Machine. Support vector machine (SVM) is a classification algorithm that plots a line that divides diverse groupings of data, and a vector is computed to optimize the line to ascertain that the closest point of each group is far away from each other as shown in Figure 16.

In our experiment with SVM, we obtained the accuracies of $75.6 \%$ and $75.0 \%$ on the training and test datasets, respectively. The accuracy on the training set is 0.756 . The accuracy on the test set is 0.750 .

The bank performance is classified as superior with a precision of $74 \%$ as illustrated in Figure 17.

3.1.7. Deep Learning. Deep learning is a subset of machine learning that involves systems that think and learn like humans using artificial neural networks. It mimics the workings of the human brain to process data (detecting objects, classifying objects, and recognizing speech) and creates patterns necessary for decision making. As illustrated in Figure 18, the algorithm builds models by making use of hidden elements in the input division to extract features, group objects, and discover useful data patterns during the 


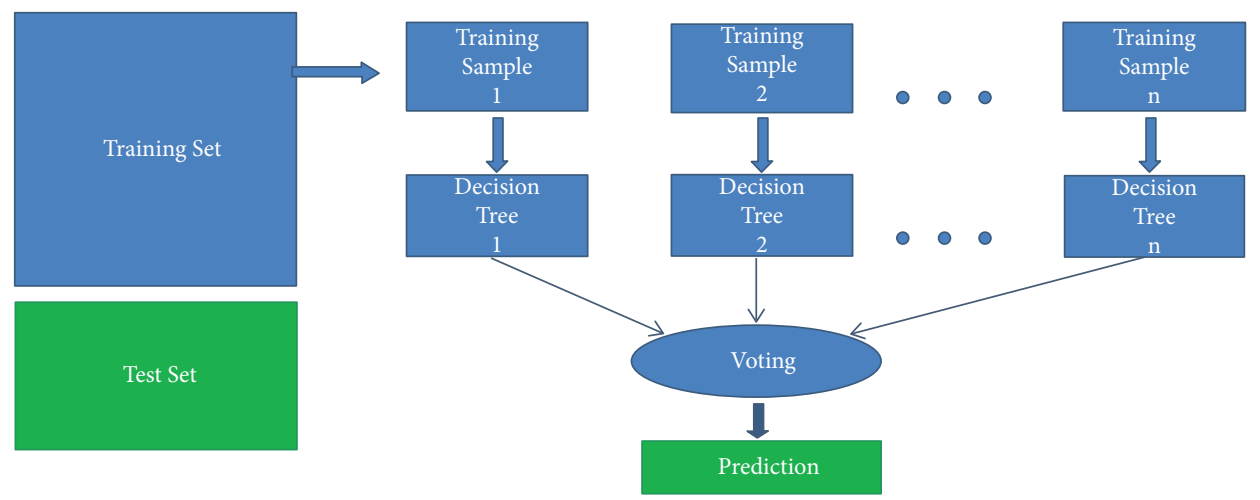

FIGURE 12: Random forest: https://www.datacamp.com/community/tutorials/random-forests-classifier-python.

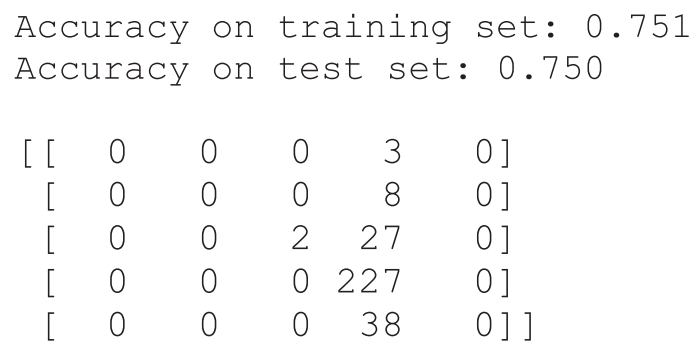

$\begin{array}{rcccr} & \text { precision } & \text { recall } & \text { fl-score } & \text { support } \\ \text { Poor } & 0.00 & 0.00 & 0.00 & 3 \\ \text { Average } & 0.00 & 0.00 & 0.00 & 8 \\ \text { Outstanding } & 1.00 & 0.07 & 0.13 & 29 \\ \text { Superior } & 0.75 & 1.00 & 0.86 & 227 \\ \text { good } & 0.00 & 0.00 & 0.00 & 38 \\ \text { accuracy } & & & & 305 \\ \text { macro avg } & 0.35 & 0.21 & 0.20 & 305 \\ \text { weighted avg } & 0.65 & 0.75 & 0.65 & 305\end{array}$

FIGURE 13: Random forest confusion matrix and accuracy results.

training process which occurs at multiple levels/layers. The computational model is made up of multiple layers, known as neural networks, where data are processed.

In this study, a deep learning algorithm was applied to the training and test datasets and obtained an accuracy of $74.2 \%$ and $75.0 \%$, respectively. The accuracy on the training set is 0.742 . The accuracy on the test set is 0.750 .

The bank performance was classified as superior with a precision of $74 \%$ as depicted in Figure 19.

3.1.8. Bayesian Classifier (Naive Bayes Model). The Naive Bayes is a classification algorithm that is based on the concept of the Bayes theorem, which is one of the fundamental theorems in probability. The Bayes theorem creates the resilience of the Naive Bayes algorithm. Data are classified using conditional probability given in the following equation. Therefore, the Naive Bayes algorithm essentially provides the probability of a record that belongs to a class, given the values of the features. This is called conditional probability.

$$
P(A \mid B)=\frac{P(B \mid A) P(A)}{P(B)},
$$

where $P(A \mid B)$ is the conditional probability of $A$ given $B$; $P(B \mid A)$ is the conditional probability of $B$ given $A ; P(A)$ is the probability of event $A ; P(B)$ is the probability of event $B$.

In our experiments with Naive Bayes, we obtained the accuracy of $73 \%$ and $80 \%$ on training and test datasets, respectively. The accuracy on the training set is 0.732 . The accuracy on the test set is 0.739 . 


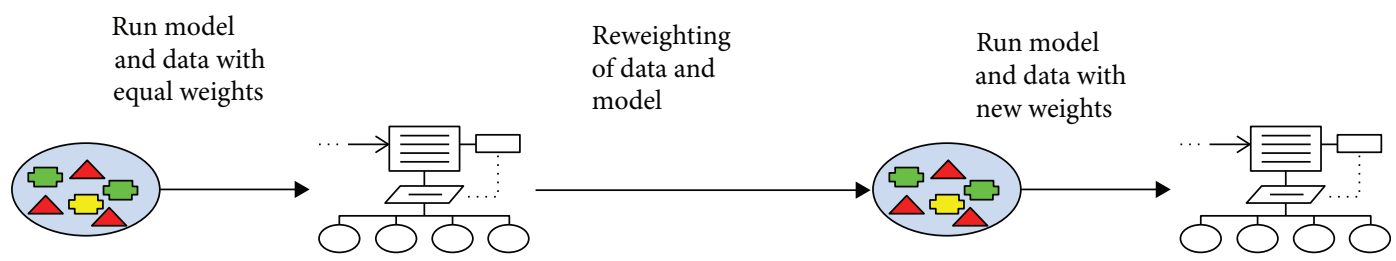

FIGURE 14: Gradient boosting classifier: https://stackabuse.com/gradient-boosting-classifiers-in-python-with-scikit-learn/.

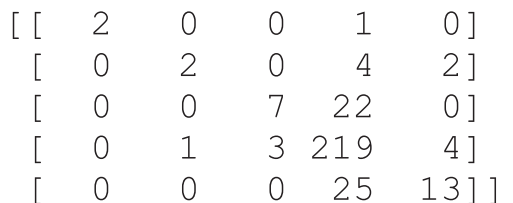

$\begin{array}{rrrrr} & \text { precision } & \text { recall } & \text { fl-score } & \text { support } \\ \text { Poor } & 1.00 & 0.67 & 0.80 & 3 \\ \text { Average } & 0.67 & 0.25 & 0.36 & 8 \\ \text { Outstanding } & 0.70 & 0.24 & 0.36 & 29 \\ \text { Superior } & 0.81 & 0.96 & 0.88 & \mathbf{2 2 7} \\ \text { good } & 0.68 & 0.34 & 0.46 & 38 \\ \text { accuracy } & & & & 305 \\ \text { macro avg } & 0.77 & 0.49 & 0.80 & 305 \\ \text { weighted avg } & 0.78 & 0.80 & 0.76 & 305\end{array}$

FIgURE 15: Gradient boosting confusion matrix and accuracy results.

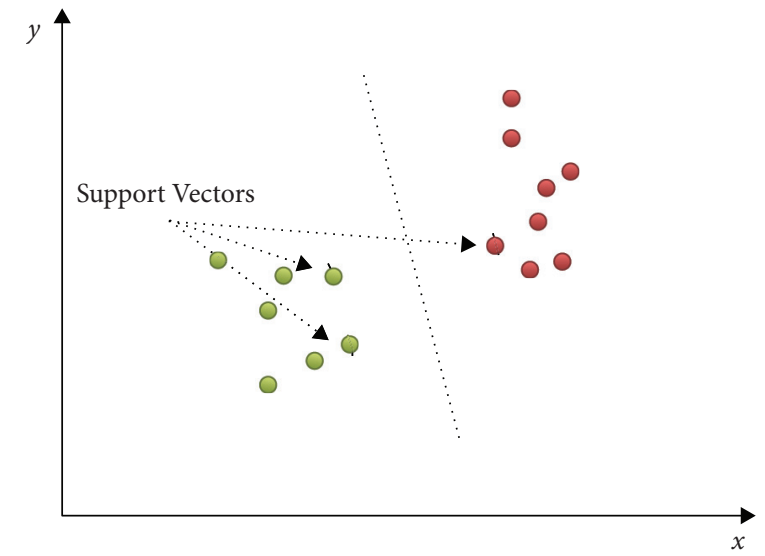

FIGURE 16: SVM hyperplane that differentiates two classes.

The bank performance was classified as superior with a precision of $79 \%$ as shown in Figure 20.

3.2. Feature Importance. The decision tree was used to evaluate the feature importance of the three features. Feature importance rates how important each feature is for the decision a tree makes. It is a number between 0 and 1 for each feature, where 0 means "not used at all" and 1 means "perfectly predicts the target." The feature importance always sums to 1 . Scikit-learn gives additional variables with the model, which illustrates the comparative importance or contribution of each feature in the prediction. It automatically computes the relevance score of each feature in the training phase and scales the significance down, so that the sum of all scores is 1 . This score is crucial as it allows you to choose the most important features and drop the least important ones for model building. In this work, the feature importance value for the evaluated features is $(0.73502277$, 0.09399781 , and 0.17097942) for employee attitude, employee behavior, and employee skills, respectively. Figure 21 illustrates that feature "employee attitude" is by far the most 


\begin{tabular}{|c|c|c|c|c|c|}
\hline [ [ & 0 & $0]$ & & & \\
\hline 0 & 0 & $0]$ & & & \\
\hline 0 & 0 & 29 & & & \\
\hline 0 & 0 & 227 & & & \\
\hline 0 & 0 & 0] ] & & & \\
\hline & & precision & recall & f1-score & support \\
\hline & Poor & 0.00 & 0.00 & 0.00 & 3 \\
\hline Ave & erage & 0.00 & 0.00 & 0.00 & 8 \\
\hline Outstan & hing & 0.00 & 0.00 & 0.00 & 29 \\
\hline Supe & erior & 0.74 & 1.00 & 0.85 & 227 \\
\hline & good & 0.00 & 0.00 & 0.00 & 38 \\
\hline $\mathrm{accu}$ & גracy & & & 0.74 & 305 \\
\hline macro & avg & 0.15 & 0.20 & 0.17 & 305 \\
\hline weighted & d avg & 0.55 & 0.74 & 0.64 & 305 \\
\hline
\end{tabular}

FIGURE 17: Support vector machine confusion matrix and accuracy results.
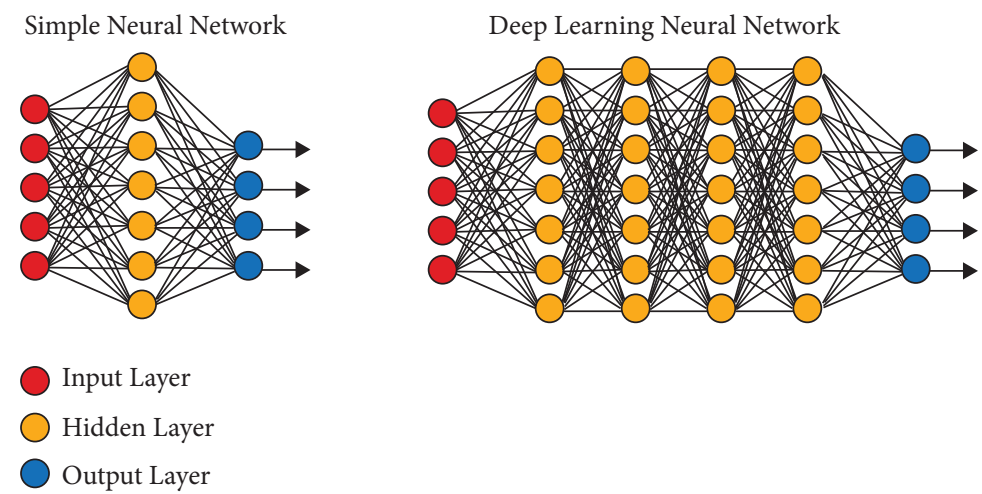

FIGURE 18: Deep learning: https://towardsdatascience.com/mnist-vs-mnist-how-i-was-able-to-speed-up-my-deep-learning-11c0787e6935.

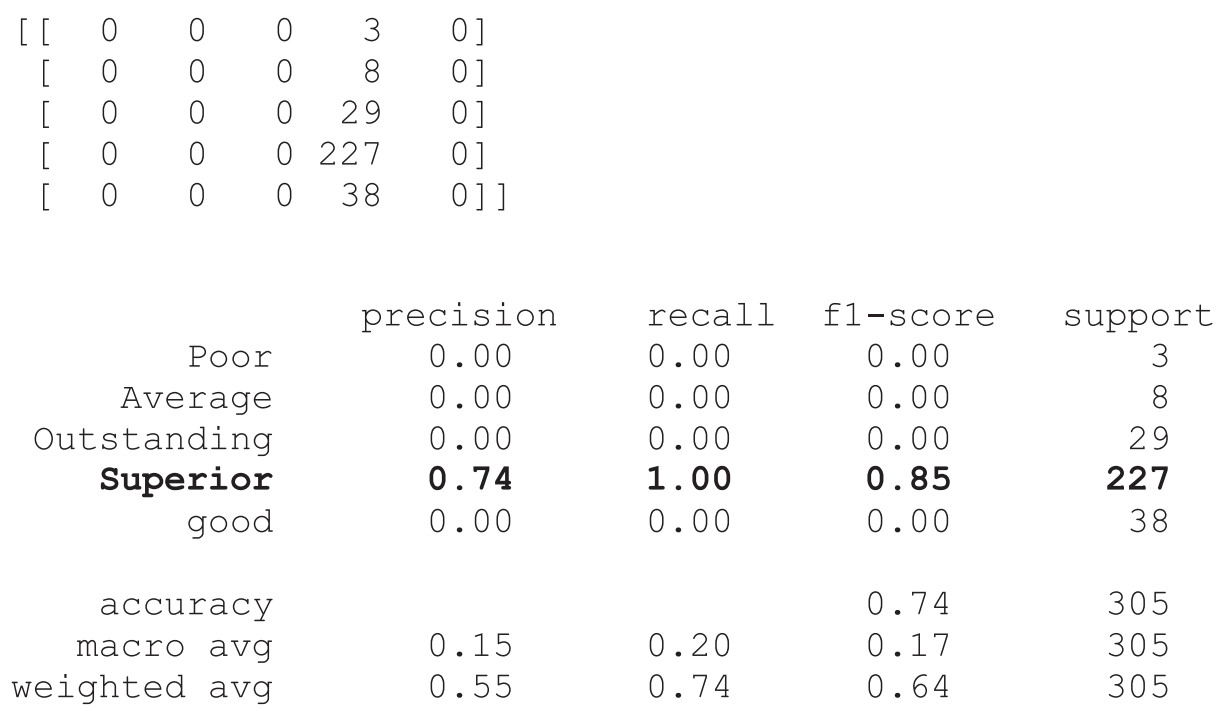

FIGURE 19: Deep learning confusion matrix and accuracy results. 


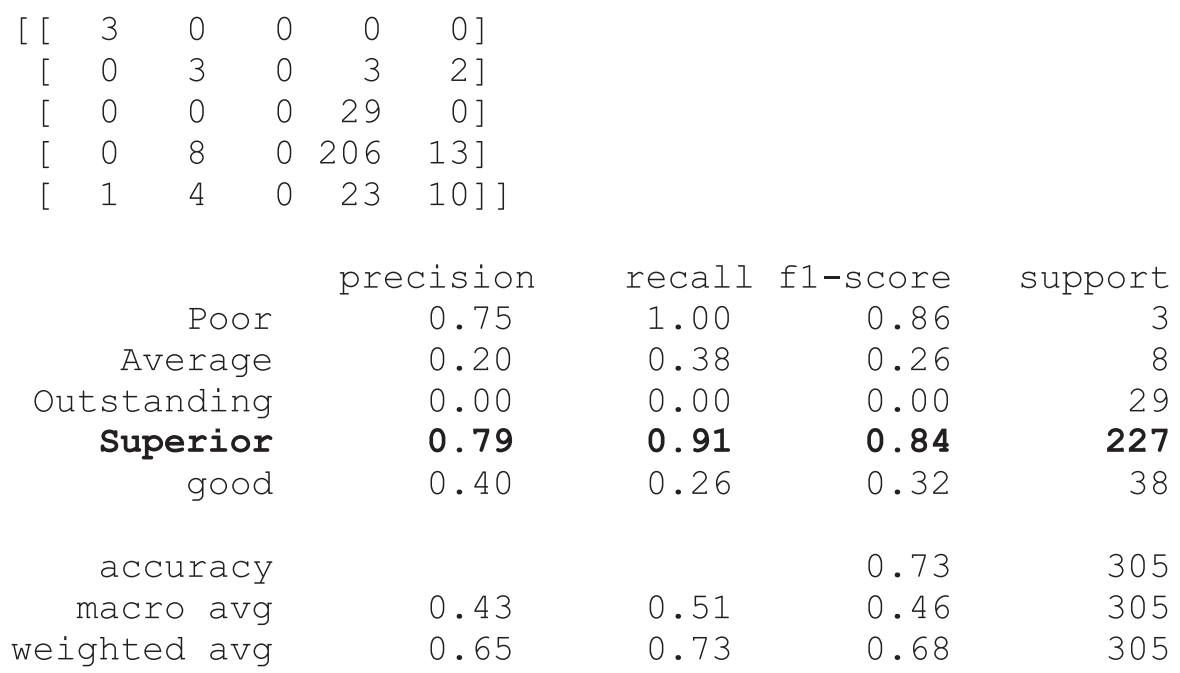

FIgURe 20: Gaussian Naïve Bayes confusion matrix and accuracy results.

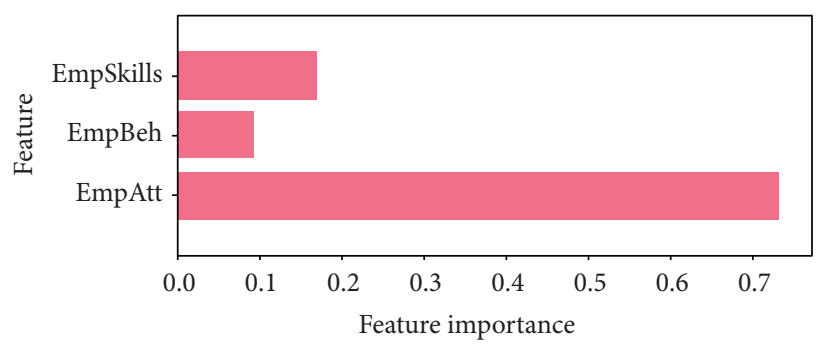

Figure 21: Feature importance.

TABLE 5: Training and test datasets accuracy.

\begin{tabular}{lccc}
\hline No. & Classifier algorithms & Accuracy on the training dataset (\%) & Accuracy on the test dataset (\%) \\
\hline 1 & K-nearest neighbor & 74.0 & 75.0 \\
2 & Logistic regression & 74.6 & 76.1 \\
3 & Decision tree & 77.9 & 71.7 \\
4 & Random forest & 75.1 & 75.0 \\
5 & Gradient boosting & 81.7 & 75.0 \\
6 & Support vector machine & 75.6 & 75.0 \\
7 & Deep learning & 74.2 & 75.0 \\
8 & Naïve Bayes & 73.2 & 73.9 \\
\hline
\end{tabular}

important feature than employee behavior and skills. Employee behavior feature can be dropped as it is less important for the bank performance model building. The focus of the banking industry should be on feature-employee attitude and try to improve on it, as this can take the bank performance from superior class to the topmost class which is "outstanding."

3.3. Classifiers and Accuracy. Table 5 illustrates the results of the eight classifiers' accuracies on split data (training and test datasets); the accuracy of the training set is similar or closer to the accuracy of the test set. This is an indication that the models built through the classifiers are efficient, there is no overfitting, and the models generalize well to new data.
Table 6 depicts the performance of the selected algorithms evaluated using the confusion matrix in Section 3.1. Table 6 illustrates how successful the classification model's predictions are, showing the crucial metrics (precision, recall, and F1-scores) for superior class because the results of all algorithms show that the bank performance is superior. Gradient boosting algorithm has the best accuracy performance as depicted in Table 6. It has the best precision of 0.81 and an F1-score of 0.88 , and its recall score of 0.96 is very close to 1.0. The recall should preferably be 1 (high) for a good classifier. Recall becomes 1 only when the numerator and denominator are equal, i.e., $\mathrm{TP}=\mathrm{TP}+\mathrm{FN}$; this also means $\mathrm{FN}$ is zero (no false negative); therefore, $\mathrm{K}$-nearest neighbor, logistic regression, random forest, support vector machine, and deep learning algorithms produce no false 
TABLE 6: Algorithms and performance measure on the classified superior class.

\begin{tabular}{lccc}
\hline Classifier algorithm & Precision & Recall & $F 1$-score \\
\hline K-nearest neighbor & 0.74 & 1.00 & 0.85 \\
Logistic regression & 0.75 & 1.00 & 0.85 \\
Decision tree & 0.77 & 0.98 & 0.86 \\
Random forest & 0.75 & 1.00 & 0.86 \\
Gradient boosting & 0.81 & 0.96 & 0.88 \\
Support vector machine & 0.74 & 1.00 & 0.85 \\
Deep learning & 0.74 & 1.00 & 0.85 \\
Naïve Bayes & 0.79 & 0.91 & 0.84 \\
\hline
\end{tabular}

negatives. Moreover, the rest of the algorithms-decision tree, gradient boosting, and Naïve Bayes also have recall scores close to 1.0 , i.e., $0.98,0.96$, and 0.91 respectively; hence, the three algorithms have very minimum false negatives. Generally, all the algorithms performed excellently having no or little false negatives. A model that produces no false positives has a precision of 1.0 , high precision relates to the low false positive rate. We obtained precisions between 0.74 and 081 for all the pretty good classifier algorithms. Furthermore, ideally in a good classifier, both precision and recall should be 1.00, which also means FP and FN are 0.00 (no false positives and negatives). Hence, we need a metric that considers both precision and recall, and an F1-score is that metric that considers both precision and recall. F1-score is the weighted average of precision and recall. A good F1score means that you have low false positives and low false negatives. An F1-score is considered perfect when it is 1.00 , while the model is a total failure when it is 0.00 . In our experiments, we obtained very high $F 1$-scores between 0.84 and 0.88 , very close to 1.00 for all the classifier algorithms (Table 6). Therefore, the performance results of the algorithms with very good precision, recall, and $F 1$-scores are an indication that the models built from the algorithms are efficient. All the models classify the bank performance as superior.

\section{Conclusion}

In this study, we employed a survey of a quantitative structured questionnaire method using efficient sampling techniques to select 305 respondents. Eight (8) different machine learning algorithms were employed to build performance models to predict the prospective performance of the Nigeria Commercial Bank Industry based on human resources outcomes (employee skills, attitude, and behavior) using Scikit-learn packages for data analytics. The results of the analysis clearly show that human resources outcomes are crucial in achieving organizational performance. The machine learning models predict the bank performance as superior with the accuracies of $74-81 \%$. The superior class has a "support" size of 227, i.e., the number of the true response samples out of the overall samples of 305. To measure the performance of the classifier algorithms, a confusion matrix was used, and the good results of the metrics such as precision, recall, and $F 1$-scores clearly show that the models are efficient. Out of the eight classifier models, gradient boosting has the best performance result. The feature importance was evaluated using the feature importance package in Scikit-learn to show the comparative importance or contribution of each feature in the prediction. Out of the three features (employee attitude, employee behavior, and employee skills), employee attitude is rated far more than others. Nigeria's bank industry needs to pay more attention to the employee attitude and improve more on it, so that the performance can be taken to the outstanding class from the current superior class.

Further work will investigate other factors and features that can also improve the performance of the commercial bank [27].

\section{Data Availability}

The data used to support the findings of this study are available upon request from the corresponding author.

\section{Conflicts of Interest}

The authors declare that they have no conflicts of interest.

\section{Acknowledgments}

The second author would like to appreciate Sol Plaatje University for the infrastructure support for this research.

\section{References}

[1] L. Cania, "The impact of strategic human resource management on organizational performance," Serial Management, vol. 17, no. 2, 2014.

[2] E. N. Caliskan, "The impact of strategic human resource management on organizational performance," Journal of Naval Science and Engineering, vol. 6, no. 2, pp. 100-116, 2010.

[3] J. O. Antwi, A. C. Opoku, A. Seth, and M. Osei-Boateng, "Assessing the human resource management practices of public banks from employees' perspective: case study of selected branches of Ghana commercial bank, kumasi," Global Journal of Human Resource Management, vol. 4, no. 1, pp. 13-30, 2016.

[4] J. Delery and N. Gupta, "Human resource management practices and organizational effectiveness: internal fit matters," Journal of Organizational Effectiveness: People and Performance, vol. 3, no. 2, pp. 139-163, 2016.

[5] E. Berkery, M. J. Morley, S. Tiernan, H. Purtill, and E. Parry, "On the uptake of flexible working arrangements and the association with human resource and organizational performance outcomes," European Management Review, vol. 14, no. 2, pp. 165-183, 2017, Summer 2017.

[6] S. O. Atiku, P. Chitakunye, and Z. Fields, "Influence of organisational learning and human resource outcomes on commercial banks' performance in Nigeria," Journal of Social Sciences, vol. 40, no. 1, pp. 9-20, 2014.

[7] V. Jayasree and R. V. S. Balan, "A review on data mining in banking sector," American Journal of Applied Sciences, vol. 10, no. 10, pp. 1160-1165, 2013.

[8] K. Chitra and B. Subashini, "Data mining techniques and its applications in banking sector," International Journal of Emerging Technology and Advanced Engineering, vol. 3, no. 8, 2013. 
[9] L. Shih-Wei, S. Yeou-Ren, C. Shih-Chi, and C. Hui-Miao, "Applying enhanced data mining approaches in predicting bank performance: a case of Taiwanese commercial banks," Expert Systems with Applications, vol. 36, pp. 11543-11551, 2009.

[10] Z. Huang, H. Chen, C.-J. Hsu, W.-H. Chen, and S. Wu, "Credit rating analysis with support vector machines and neural networks: a market comparative study," Decision Support Systems, vol. 37, no. 4, pp. 543-558, 2004.

[11] I. Gede Riana, G. Suparna, I. Gusti Made Suwandana, S. Kot, and I. Rajiani, "Human resource management in promoting innovation and organizational performance," Problems and Perspectives in Management, vol. 18, no. 1, pp. 107-118, 2020.

[12] G. M. Pradhan, "Impact of human resource management practices on behavioural outcomes of financial institutions of Nepal," The Batuk, vol. 6, no. 1, pp. 17-27, 2020.

[13] M. Alatailat, H. Elrehail, and O. L. Emeagwali, "High-performance work practices, organizational performance, and strategic thinking," International Journal of Organizational Analysis, 2019.

[14] P. Maroufkhani, M. L. Tseng, M. Iranmanesh, W. K. W. Ismail, and H. Khalid, "Big data analytics adoption: determinants and performances among small to mediumsized enterprises," International Journal of Information Management, vol. 54, Article ID 102190, 2020.

[15] M. R. Islam and M. A. Habib, "A data mining approach to predict prospective business sectors for lending in retail banking using decision tree," International Journal of Data Mining and Knowledge Management Process, vol. 5, no. 2, pp. 13-22, 2015.

[16] I. G. N. N. Mandala, C. B. Nawangpalupi, and F. R. Praktikto, "Assessing credit risk: an application of data mining in a rural bank," Procedia Economics and Finance, vol. 4, pp. 406-412, 2012.

[17] T. F. Bahari and M. S. Elayidom, "An efficient CRM-data mining Framework for the prediction of customer behaviour," Procedia Computer Science, vol. 46, pp. 725-731, 2015.

[18] D. Kabakchieva, "Student performance prediction by using data mining classification algorithms," International Journal of Computer Science and Management Research, vol. 1, no. 4, pp. 2278-733X, 2012.

[19] D. Kabakchieva, "Predicting student performance by using data mining methods for classification," Cybernetics and Information Technologies, vol. 13, no. 1, pp. 61-72, 2013, Print ISSN: 1311-9702; Online ISSN: 1314-4081.

[20] http://www.zentut.com/data-mining/Data Mining Tutorial.

[21] S. O. Atiku, "The relationship between organizational culture and performance: a case of the banking sector in Nigeria," Ph.D. thesis, University of KwaZulu-Natal, Durban, South Africa, 2014.

[22] Group, Fitch, 2011 Fiscal, FIMALAC, Paris, France, 2012.

[23] A. Onan, S. Korukoğlu, and H. Bulut, "Ensemble of keyword extraction methods and classifiers in text classification," Expert Systems with Applications, vol. 57, pp. 232-247, 2016.

[24] A. Onan, "Classifier and feature set ensembles for web page classification," Journal of Information Science, vol. 42, no. 2, pp. 150-165, 2016.

[25] A. Onan, "Biomedical text categorization based on ensemble pruning and optimized topic modelling," Computational and Mathematical Methods in Medicine, vol. 2018, Article ID 2497471, 22 pages, 2018.

[26] T. O. Ayodele, "Types of machine learning algorithms," New advances in machine learning, vol. 3, pp. 19-48, 2010.
[27] A. Onan, "Biomedical text categorization based on ensemble pruning and optimized topic modelling," Computational and Mathematical Methods in Medicine, vol. 2018, Article ID 2497471, 22 pages, 2018. 